\title{
Early Syringomyelia in Tubercular Meningitis: A Rare Occurrence
}

\author{
Sanjay Pandey ${ }^{\mathrm{a}}$, Rajeev Nayak ${ }^{\mathrm{a}, \mathrm{b}}$, Man Mohan Mehndiratta
}

\begin{abstract}
Tubercular meningitis (TBM) can have varied early and delayed complications involving brain or spinal cord. Although syringomyelia can occur sometimes as a late complication of tubercular meningitis, its occurrence in early stages of TBM is very rare. There are only two published case reports of syringomyelia in acute stage of TBM. We report a patient with tubercular meningitis who developed syringomyelia in early disease course. A 26-year-old female presented with history of fever, severe headache and vomiting for last two and half months. Patient's history and clinical examination was suggestive of chronic meningitis with raised intracranial pressure. Cerebrospinal fluid analysis showed evidence of TBM. Patient was started on antitubercular drugs and during treatment course she developed acute onset motor weakness of all four limbs. Magnetic resonance imaging (MRI) showed long segment syrinx extending from $\mathrm{C} 3$ to $\mathrm{T} 3$ region. After thecoperitoneal shunting there was improvement in motor power.
\end{abstract}

Keywords: Tubercular meningitis; Syringomyelia; Arachnoiditis

\section{Introduction}

Tubercular meningitis (TBM) is the most common form of central nervous system tuberculosis and one of the leading cause of morbidity and mortality in developing world. TBM can have varied early and delayed complications including hydrocephalus, cranial nerve palsies, vasculitic infarction, arachnoiditis and tuberculoma involving brain or spinal cord. Sometimes syringomyelia can also occur as a late

Manuscript accepted for publication January 30, 2013

${ }^{a}$ Department Of Neurology, G.B. Pant Hospital, Delhi University, New Delhi, India

${ }^{\mathrm{b}}$ Corresponding author: Rajeev Nayak, Academic block, G.B. Pant Hospital, J.L.N. Marg, New Delhi-2, Postal index-110002 India.

Email: nayakdrrajeev79@yahoo.com

doi: http://dx.doi.org/10.4021/jnr172w complication of tubercular meningitis [1]. Although syrinx formation in early stage of TBM is very rare. There are only two published case reports of syringomyelia in acute stage of TBM $[2,3]$. Here we report a patient with tubercular meningitis who developed syringomyelia in early course of illness.

\section{Case Report}

A 26-years-old female presented with history of fever, severe headache and projectile vomiting for last two and half months. Clinical examination revealed bilateral papilledema and signs of meningeal irritation. Patient was fully conscious, alert and oriented. Neurological examination including cranial nerves and motor system examination was also unremarkable. Her complete hemogram, blood glucose, blood urea, serum creatinine, serum bilirubin and transaminase levels were within normal range. Contrast enhanced CT scan head was normal (Fig. 1A). Cerebrospinal fluid (CSF) analysis revealed leukocyte count 800 cells/cubic $\mathrm{mm}$ with lymphocyte count $90 \%$, glucose $26 \mathrm{mg} \%$ (Corresponding blood sugar $90 \mathrm{mg} \%$; ratio 1/3) and protein level of $226 \mathrm{mg} \%$. CSF acid fast Bacilli (AFB) staining was negative. Cryptococcus antigen and India ink preparation for Cryptococcus was also negative. In past, she had low back pain 12 years back for which she was diagnosed as Pott's spine and received antitubercular treatment. She did not have any neurological deficit during that period and remained asymptomatic till presenting symptoms. Any follow up neuroimaging was not done in this period as patient was absolutely asymptomatic. Considering clinical features of chronic meningitis and CSF picture suggestive of tubercular meningitis, she was started on first line antitubercular drugs, steroids and antiedema drugs. During hospitalisation, on 15th day she developed two episodes of generalised tonic clonic seizures and started on tablet phenytoin. After initiation of treatment patient showed improvement in headache for few days, but after 2 weeks she developed worsening of headache and vomiting. Contrast enhanced CT scan head was repeated which revealed communicating hydrocephalus and meningeal enhancement (Fig. 1B). After 7 days she developed weakness of all four limbs. She also developed inability to control micturition. 


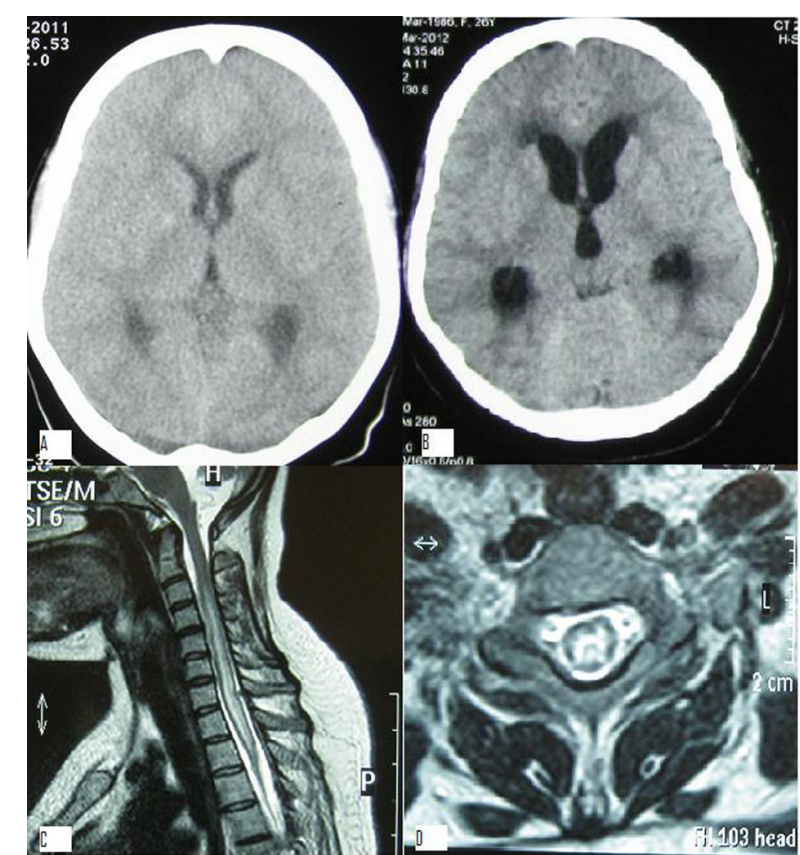

Figure 1. A). Contrast enhanced CT scan head before admission which was normal; B). Contrast enhanced CT scan head done after admission showing communicating hydrocephalus; C). Magnetic resonance imaging (MRI) T2 weighted sagittal image showing intramedullary hyper intensity extending from C3 to T3 level suggestive of syrinx; D). MRI T2 weighted coronal section through $\mathrm{C} 6$ level showing intramedullary hyperintensity suggestive of syringomyelia.

Motor system examination revealed hypotonia of all four limbs. Motor weakness was more in upper limbs (MRC grade 1/5) in comparison to lower limbs (MRC grade 2/5). Deep tendon reflexes were absent in upper limbs and brisk in lower limbs. Planter reflex were extensor bilaterally. MRI spine showed long segment syrinx extending from C6 to T3 level (Fig. 1C, D). Patient underwent thecoperitoneal shunt and patient showed some improvement in motor power (upper limb 2/5, lower limbs 3/5 MRC grade) after two weeks of shunt procedure.

\section{Discussion}

Syringomyelia is a known late complication of TBM. After searching English literature we could find out only 26 case reports regarding syrinx formation after tubercular meningitis. Usually the interval between TBM and syrinx formation varies from 7 - 28 years [3]. In our case syringomyelia developed in early stages of TBM during the treatment, which is very rare. Daif et al first time described two cases of syringomyelia in early course of tubercular meningitis (after 11 days and 6 weeks) [2]. Exact pathogenesis of syrinx formation in TBM is not known. However there are several proposed mechanisms in the syrinx formation after tubercular meningitis. Syrinx formation may be secondary to either an obliterative endarteritis causing ischemic injury or softening of spinal cord or may be because of post inflammatory scarring $[4,5]$. Focal scarring due to inflammatory vasculitis may lead to blockage of the CSF circulation, thus forcing CSF into the central canal of the spinal cord through VirchowRobin spaces. Obliteration of these perivascular spaces may also occur and lead to focal cystic dilatations of the spinal cord that eventually coalesce to form syrinx. The cause of rapidly progressive myelopathy in TBM has been attributed to vascular thrombosis of the spinal cord vessels. Spinal subarachnoid CSF space obstruction leads to a relative increase in the pressure inside the spinal cord distal to the block. This produces a pressure gradient between inside and outside the spinal cord in the segment distal to the blockage. Repetitive production of this pressure gradient at each CSF pulse causes leakage of CSF from the central canal, leading to interstitial oedema and the formation of a syrinx. Tubercular endarteritis producing softening of the cord, spinal subarachnoid space scarring leading to reduction in the compliance of the subarachnoid compartment and the patent Virchow-Robin spaces in the spinal cord providing a conduit for the CSF to enter into the central canal are three basic mechanisms in the formation of syrinx in TBM. In our case we postulated that in addition to spinal cord vascular thrombosis due to tubercular arteritis, development of communicating hydrocephalus and acute rise in intracranial tension contributed in pathogenesis of syrinx formation.

\section{Conclusion}

In a patient with tubercular meningitis development of new neurological manifestations like motor weakness should raise the possibility of syrinx formation after excluding common complications like vascultic stroke or spinal arachnoiditis.

\section{Disclosure}

None.

\section{Conflict of Interest}

None.

\section{Abbreviations}

TBM: Tubercular Meningitis; CSF: Cerebrospinal fluid; MRC: Medical Research Council 


\section{References}

1. Kaynar MY, Kocer N, Gencosmanoglu BE, Hanci M. Syringomyelia--as a late complication of tuberculous meningitis. Acta Neurochir (Wien). 2000;142(8):935938; discussion 938-939.

2. Daif AK, al Rajeh S, Ogunniyi A, al Boukai A, al Tahan A. Syringomyelia developing as an acute complication of tuberculous meningitis. Can J Neurol Sci. 1997;24(1):73-76.
3. Moghtaderi A, Alavi-Naini R, Rahimi-Movaghar V. Syringomyelia: an early complication of tuberculous meningitis. Trop Doct. 2006;36(4):254-255.

4. Pepin B, Frenay J, Goldstein B, Haguenau M, Le Besnerais Y, Guillard A, Boudin G. [Syringomyelic syndrome with tuberculous meningitis (apropos of 4 cases)]. Rev Neurol (Paris). 1977;133(12):697-708.

5. Caplan LR, Norohna AB, Amico LL. Syringomyelia and arachnoiditis. J Neurol Neurosurg Psychiatry. 1990;53(2):106-113. 